\title{
Effect of Lubrication on the Changes in Tensile Properties of Cotton Sewing Thread at Different Stages of Sewing
}

\author{
Vinay Kumar Midha ${ }^{a, *}, \quad$ Sushma Verma ${ }^{\mathrm{b}}$ \\ a Professor, Department of Textile Technology, Dr. B R Ambedkar National Institute of Technology, \\ Jalandhar, Punjab (INDIA)-144011 \\ ${ }^{\mathrm{b}}$ Research Scholar, Department of Textile Technology, Dr. B R Ambedkar National Institute of \\ Technology, Jalandhar, Punjab (INDIA)-144011
}

\begin{abstract}
Friction forces play a very important role in performance of sewing thread during the sewing process. Various finishes are applied over sewing threads to reduce the friction forces, which help in masking the basic properties of sewing thread and reduce the yarn to metal friction and yarn to yarn friction. In this paper, the effect of lubrication (\%) on tensile properties of sewing thread is measured at four sewing stages: before sewing, after dynamic loading at the tension regulator, after passage through the needle and fabric assembly, and after bobbin thread interaction. It is found that as the lubrication per cent increases friction coefficient decreases, in general. Due to this, the loss in tensile properties of the sewing threads during sewing decrease. Tenacity loss decreases during all sewing stages, as the lubrication percentage increases.
\end{abstract}

Keywords: Lubrication; Sewing Stages; Tenacity

\section{Introduction}

During sewing at high speeds, needle thread is subjected to repeated tensile stresses at very high rates. The value of these stresses depends on the sewing speed, machine tension settings, stitch density and sewing thread properties. Due to these stresses, thread shows a negative impact on sewing and its functional characteristics; therefore, significant reduction in the sewing thread strength occurs [1]. A number of researchers observed that there could be $30-40 \%$ strength reduction in the cotton thread after sewing, which is due to the structural disintegration, dynamic loading, abrasion and bending deformation [2-4].

The friction coefficient of sewing threads is an important factor for the production process and performance of the garments [5]. Sewing threads develop friction when they pass over various machine parts during the garment manufacturing process. The amount of friction between guiding elements and sewing threads depends on the surface treatment of the thread [5-6]. Various

\footnotetext{
${ }^{*}$ Corresponding author.

Email address: midhav@rediffmail.com (Vinay Kumar Midha).
} 
lubricants are applied over sewing threads to provide a controlled level of friction by masking the thread surface [7-8]. In a recent publication, it is reported that the amount and type of the lubricant applied have a profound effect on frictional properties of the thread. Friction can be 200 times lower for thread with lubrication, as compared to thread without lubrication [9-10]. It is found that most of the researchers have focused on the effect of lubrication on frictional properties of the thread but no study has reported the effect of lubrication on sewing thread strength before sewing and during various stages of sewing. In this paper, the effect of different levels of lubrication has been studied on tensile properties of the threads as the thread progresses through various stages of sewing.

\section{Experimental}

Cotton sewing thread of 14 Tex is used to produce three different samples by varying lubrication (\%) as shown in Table 1. Silicone and wax finish is applied over the threads as a lubricant. The physical properties of the threads are also shown in Table 1.

Table 1: Physical properties of sewing threads

\begin{tabular}{lccccccc}
\hline \multirow{2}{*}{$\begin{array}{c}\text { Type of } \\
\text { thread }\end{array}$} & $\begin{array}{c}\text { Twist } \\
\text { direction }\end{array}$ & $\begin{array}{c}\text { Twist (Turns } \\
\text { per cm) }\end{array}$ & $\begin{array}{c}\text { Linear } \\
\text { density (Tex) }\end{array}$ & $\begin{array}{c}\text { Lubrication } \\
(\%)\end{array}$ & $\begin{array}{c}\text { Tenacity } \\
(\mathrm{cN} / \text { Tex })\end{array}$ & \multicolumn{2}{c}{ Friction coefficient } \\
\cline { 7 - 8 } & & & & 3 & 35.76 & 0.378 & 0.366 \\
\multirow{2}{*}{ Cotton } & \multirow{2}{*}{20} & \multirow{2}{*}{16} & 4 & 42.78 & 0.365 & 0.354 \\
& & & & 5 & 36.8 & 0.376 & 0.332 \\
\hline
\end{tabular}

Tensile testing of threads (before and after sewing) is performed at a gauge length of $250 \mathrm{~mm}$ on Universal testing machine as per ASTM standard D2256. Thirty tests are carried out and the error at the $95 \%$ confidence interval is found to be less than $4 \%$. Brother industrial lockstitch sewing machine is run at a speed of 4000 stitches/min, to produce a seam on three layers of rib knitted fabric with stitch density of 9 stitches $/ \mathrm{cm}$. The same type of thread is used for needle and bobbin thread to prepare the seams. Sewing thread tensile properties are measured at four sewing stages (S1, S2, S3, and S4) as described in literature [11]. The difference in tensile property between any two stages is due to the stresses acting during that stage as shown below;

- stages S1 and S2 - dynamic loading;

- stages S2 and S3 - passage through needle and fabric assembly;

- stages S3 and S4 - bobbin thread interaction

The change (\%) and the contribution (\%) of the stresses in total change in the tensile property at different stages are calculated from the following expressions:

$$
\begin{aligned}
& \text { Change (\%) }=\frac{T_{n}-T_{n-1}}{T_{n-1}} \\
& \text { Contribution }(\%)=\frac{T_{n}-T_{n-1}}{T_{1}-T_{4}}
\end{aligned}
$$

where $T_{n}$ is the tensile property at different sewing stages, with $n=2,3$, and 4 corresponding to sewing stage S2, S3, and S4, respectively. 
$T_{n-1}$ is the tensile property of the thread of the previous stage. Negative (-) change (\%) shows the loss in tensile property, whereas a positive change (\%) indicates the gain in the tensile property.

Yarn to yarn co-efficient of friction measurement is carried out by Fixed Yarn-Body Capstan method according to ASTM D 3412-13. A length of yarn is passed at a known speed over the stationary surface making a specified wrapping angle and the coefficient of friction is calculated by using the Capstan formula as follows:

$$
\mu=\frac{1}{2 \theta} \ln \frac{T}{W}
$$

where, $\mu=$ Co-efficient of yarn-yarn friction

$\theta=$ Wrapping angle of sliding yarn over the stationary surface

$T=$ Mean output tension, $\mathrm{N}$

$W=$ Input tension, $10 \mathrm{mN} / \mathrm{tex}$

Yarn to metal friction is measured according to the ASTM D 3108-01 using Lawson Hemphill's friction tester. To carry out this test, the thread is guided through the tension disc and guides. Input and output tensions are measured by a suitable tension gauge which produces an electrical signal. The ratio of output to input tension is established directly and the coefficient of friction indicated on a scale or display.

\section{Result and Discussion}

Tensile properties of the needle thread are measured at different sewing stages for all the sewing threads with different lubrication percentage. The change in tensile properties and contribution of different zones (S2, S3, and S4) have been calculated using equations 1-2. Table 2 shows the change in tenacity, breaking elongation and initial modulus in different zones of the thread's passage through the machine, along with the contribution of each zone.

\subsection{Tenacity \& Breaking Elongation}

As the lubrication percentage increases, the tenacity of threads increases marginally. However, the increase in tenacity is not statistically significant. However, elongation percentage of the threads increases and then decreases as lubrication percentage increases. A progressive decrease in tenacity is observed as the thread passes through various sewing stages. Fig. 1 shows the contribution of dynamic loading, passage through needle and fabric assembly, and bobbin thread interaction in total loss in tenacity for all threads having different lubrication percentage. It is observed that bobbin thread interaction ( $\mathrm{S} 4$ zone) is the largest contributor to tenacity loss of the threads followed by the dynamic loading ( $\mathrm{S} 2$ zone), and passage through needle and fabric assembly (S3 zone). Needle and bobbin thread interaction is the major contributor towards elongation loss (Fig. 2), in confirmation with the previous studies [11-12]. Total loss in tenacity for all threads decreases from $29.73 \%$ to $14.16 \%$ with increase in lubrication percentage. Elongation loss also reduces significantly with the increase in lubrication percentage. 
Table 2: Changes in tensile properties of sewing thread at different stages of sewing

\begin{tabular}{|c|c|c|c|c|c|c|c|c|c|c|}
\hline \multicolumn{2}{|c|}{ Tensile properties } & \multicolumn{3}{|c|}{ Tenacity (cN/Tex) } & \multicolumn{3}{|c|}{ Elongation (\%) } & \multicolumn{3}{|c|}{ Initial modulus (cN/Tex) } \\
\hline $\begin{array}{l}\text { Sewing } \\
\text { Stages }\end{array}$ & Lubrication (\%) & 3 & 4 & 5 & 3 & 4 & 5 & 3 & 4 & 5 \\
\hline $\mathrm{S} 1$ & Mean & 37.54 & 37.51 & 38.55 & 4.19 & $5.23^{*}$ & $4.58^{*}$ & 1304 & $781^{*}$ & $723^{*}$ \\
\hline \multirow[t]{3}{*}{ S2 } & Mean & 35.02 & $36.57^{*}$ & 37.37 & 4.00 & $5.01^{*}$ & $4.25^{*}$ & 821 & 830 & $805^{*}$ \\
\hline & Change (\%) & -6.71 & -2.50 & -3.06 & -4.44 & -4.05 & -6.99 & -37.04 & 6.27 & 11.34 \\
\hline & Contribution (\%) & -22 & -16.3 & -21.6 & -9.26 & -12.64 & -25.4 & -69.09 & 77.77 & 167.34 \\
\hline \multirow[t]{3}{*}{ S3 } & Mean & 33.57 & $35.40^{*}$ & 36.70 & 3.34 & $4.40^{*}$ & $3.87^{*}$ & 798 & $844^{*}$ & 840 \\
\hline & Change (\%) & -4.14 & -3.19 & -1.80 & -16.5 & -12.17 & -8.94 & -2.80 & 1.68 & 4.34 \\
\hline & Contribution (\%) & -12.9 & -20.24 & -12.27 & -32.19 & -35.05 & -29.23 & -3.29 & 22.22 & 71.42 \\
\hline \multirow[t]{4}{*}{$\mathrm{S} 4$} & Mean & 26.38 & $31.73^{*}$ & 33.09 & 2.14 & $3.49^{*}$ & $3.28^{*}$ & $605^{*}$ & $719^{*}$ & $772^{*}$ \\
\hline & Change (\%) & -21.4 & -10.34 & -9.82 & -35.65 & -20.51 & -15.19 & -24.18 & -14.81 & -8.09 \\
\hline & Contribution (\%) & -64.4 & -63.49 & -66.11 & -58.53 & -52.29 & -45.38 & -27.61 & -198.41 & -138.77 \\
\hline & Total loss $(\%)$ & -29.73 & -15.40 & -14.16 & -48.92 & -33.26 & -28.38 & -53.60 & -7.93 & 6.77 \\
\hline
\end{tabular}

negative sign shows the loss in tensile properties

shows the significant difference of means at a $95 \%$ confidence interval from the previous lubrication $\%$

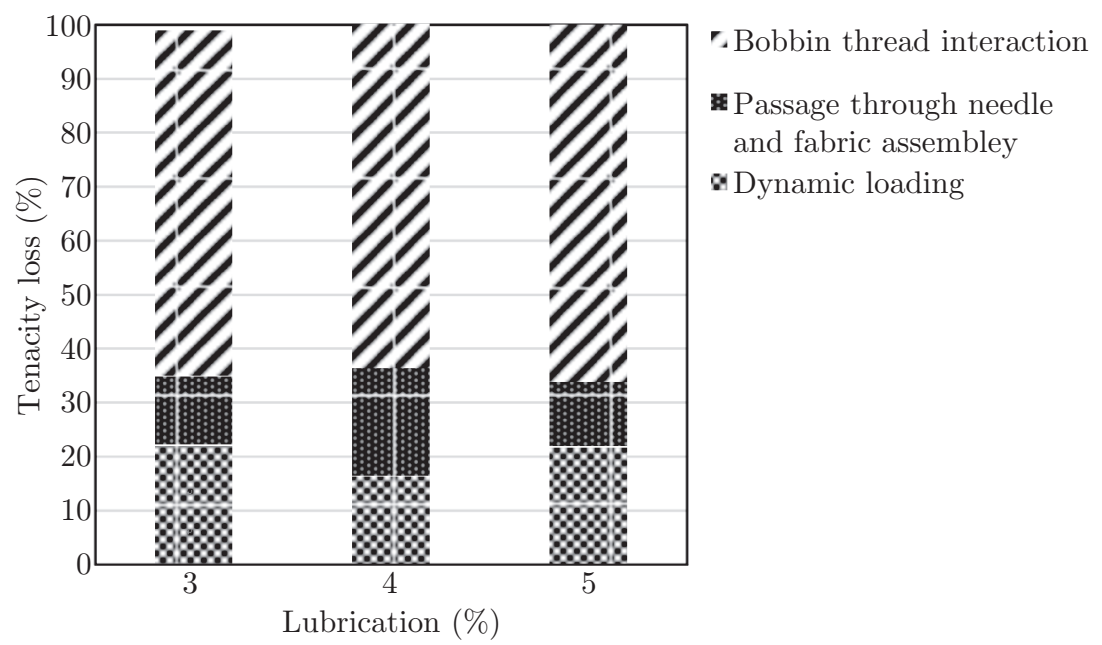

Fig. 1: Contribution of dynamic loading, passage through needle and fabric assembly and bobbin thread interaction in tenacity loss of threads

In S2 zone, as the lubrication percent increases, overall tenacity loss for all the sewing threads decreases from $6.71 \%$ to $3.06 \%$. The decrease in tenacity loss is statistically significant as lubrication increases from $3 \%$ to $4 \%$. However the decrease is statistically insignficant as lubrication is increased from $4 \%$ to $5 \%$. In this zone, thread undergoes various stresses and strains such as dynamic loading when it moves from cone to seam. Cotton has convoluted cross section, and therefore there is scope of readjustment of fibres during dynamic loading. At higher level of lubrication, silicon oil enters into the structure and causes fibre slippage within the structure [10]. Fibre slippage facilitates the rearrangement of fibres during dynamic loading leading to 


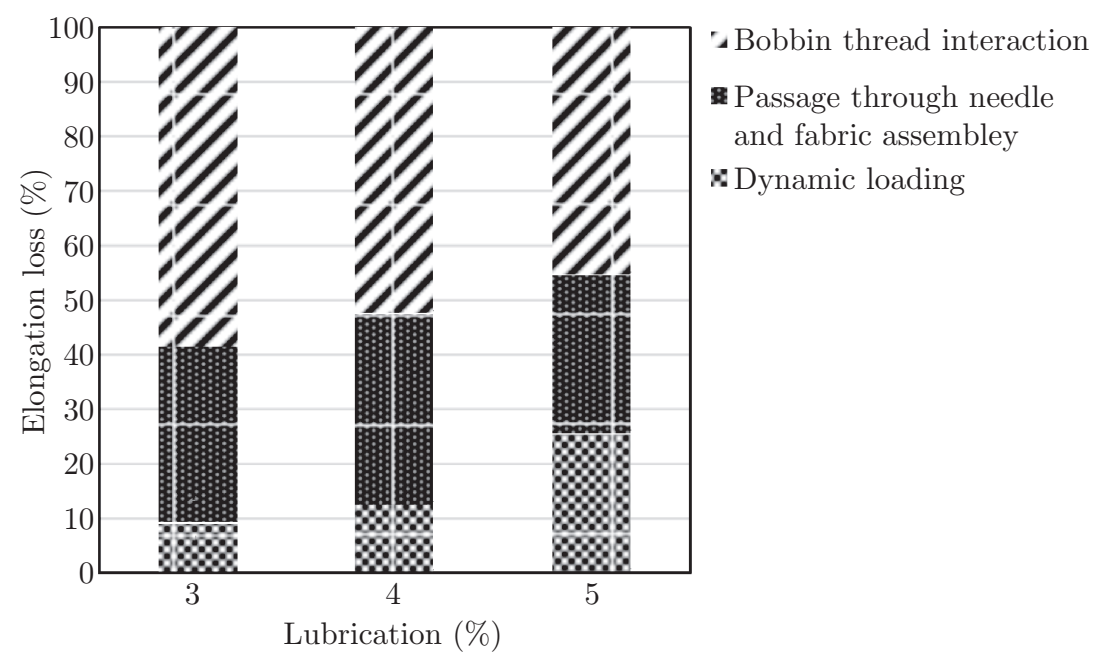

Fig. 2: Contribution of dynamic loading, passage through needle and fabric assembly and bobbin thread interaction in elongation loss of threads

improved load bearing capacity of the thread and therefore tenacity loss decreases. However, elongation loss shows opposite trend. As the lubrication percentage increases, elongation loss percent increases from $4.44 \%$ to $6.99 \%$ percent during S2 zone. At higher lubrication percentage, due to rearrangement of fibres, the straightened fibres in the yarn structure do not contribute to elongation.

Passage through the needle and fabric assembly (S3 zone) is the smallest contributor towards tenacity loss (Fig. 1). With increased lubrication, tenacity loss in this zone further reduces from $4.14 \%$ to $1.80 \%$. In S3 zone, thread repeatedly passes through needle eye and fabric assembly due to which tenacity loss occurs. When lubrication is applied, it provides a film outside the surface, due to which coefficient of friction decreases, in general (Table 1). Elongation loss also decreases from $16.5 \%$ to $8.94 \%$ as lubrication percent increases. Decrease in coefficient of friction leads to lower damage through abrasion and bending during the passage through needle and fabric assembly.

Bobbin thread interaction is the largest contributor towards tenacity loss (Fig. 2). In this zone (S4), needle thread interacts with the bobbin thread. Improved lubrication helps in the reduction of yarn to yarn friction (Table 1), which enhances the mobility of the yarn and reduces the possibility of needle thread damage. Hence, lower loss in tenacity is observed in S4 zone. Elongation loss also decreases from $48.92 \%$ to $28.38 \%$ due to the similar reason.

\subsection{Initial Modulus}

As lubrication percentage increases, initial modulus decreases significantly. A sharp decrease in initial modulus is observed as the lubrication percentage increases from $3 \%$ to $4 \%$. Sewing thread with $3 \%$ lubrication shows significant initial modulus loss at all sewing stages as shown in Fig. 3. Lower level of lubrication provides higher yarn to metal and yarn to yarn friction, which cause higher damage to the thread during dynamic loading (S2), passage through needle and fabric assembly (S3) and bobbin thread interaction (S4). The rubbing of yarns against various machine parts and dynamic loading causes the fibers to protrude from the yarn surface, leading to loss in initial modulus. 


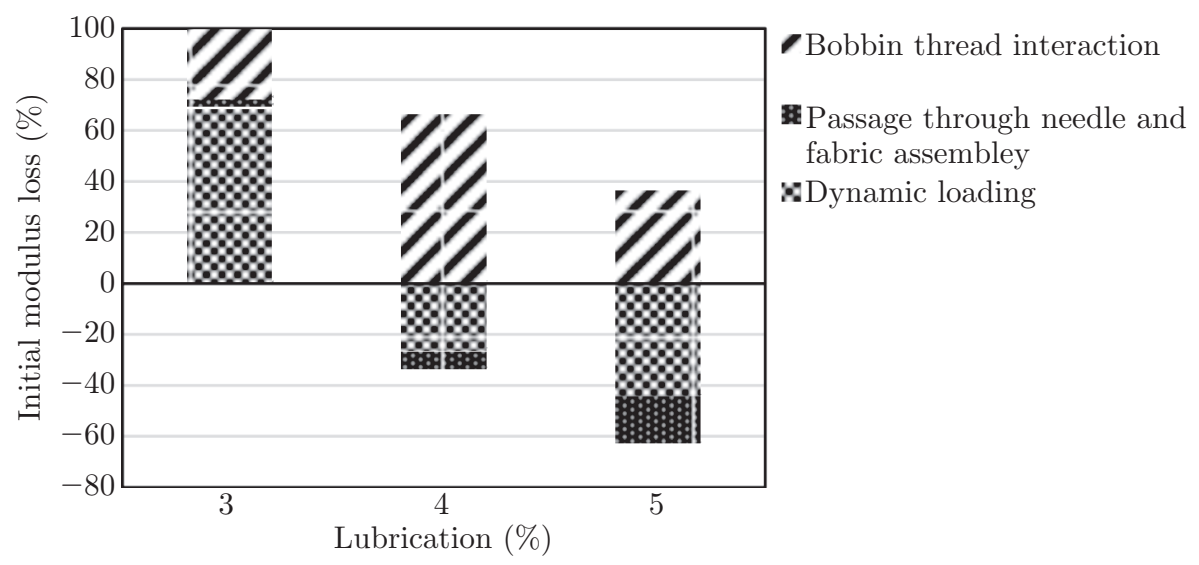

Fig. 3: Contribution of dynamic loading, passage through needle and fabric assembly and bobbin thread interaction in initial modulus loss of threads

As the lubrication increases to $4 \%$ and 5\%, significant gain in initial modulus is observed in S2 and S3 zone (Fig. 3), which is in confirmation to the earlier studies. In S2 zone, gain in initial modulus is observed because fiber slippage helps fibers to rearrange themselves and increase the load bearing capacity of threads. Moreover, the higher lubrication reduces the coefficient of friction. As the thread moves from S3 to S4 zone, initial modulus loss decreases with improved level of lubrication. Higher amount of lubricant reduces the contact area between the materials and provides smooth surface to the yarn which causes decrease in yarn to yarn friction coefficient. Hence, contribution of initial modulus loss decreases.

\section{Conclusion}

The lubrication over the sewing thread is a crucial factor to determine the properties of the sewing threads, particularly in improving the sewability. Lubricant helps in reducing the coefficient of friction between yarn to metal and yarn to yarn. The major loss in tenacity for the threads in S4 zone can be reduced significantly from $21.4 \%$ to $9.82 \%$ by increasing the lubrication percent. A marginal decrease in tenacity loss is observed in S2 and S3 zone also. Total loss in tenacity is reduced from $29.73 \%$ to $14.16 \%$ with the help of increased lubrication percentage. According to the study, $4 \%$ lubrication is suggested to get minimum tenacity loss for sewing threads. After that, if lubrication percentage is increased, insignificant change in tenacity loss is observed in all zones.

\section{References}

[1] R. M. Crow, N. H Chamberlain, The performance of sewing threads in industrial Sewing Machines, Clothing Institute Technological Report. 21 (1969).

[2] G. Sundaresan, P. K. Hari, K. R Salhotra, Strength reduction in sewing threads during high speed sewing in an industrial lockstitch machine: Part I- mechanism of thread strength reduction, International Journal of Clothing Science and Technology. 9(1997), 334-345.

[3] G. Sundaresan, P. K. Hari, K. R Salhotra, Strength reduction in sewing threads during high 
speed sewing in an industrial lockstitch machine: Part II- effect of thread and fabric properties, International Journal of Clothing Science and Technology. 10(1998), 64-79.

[4] D. Z. Lojen, J. Gersak, Determination of the sewing thread friction coefficient. International Journal of Clothing Science and Technology. 15(2003), 241-249.

[5] G. Balc1, V. Su-lar, Yarn friction properties: importance and test methods, J Textil Eng: 16(2009), 6-15.

[6] S. Altas and H. Kadoğlu, Yarn to yarn and yarn to metal friction in relation on to some properties of yarn, Tekstil ve Mühendis. 16(2009), 1-5.

[7] E. Yukseltan. Investigation of the effect of lubricant application on sewing threads performance properties and on seam breaking strength. MSc Thesis, Uludag University, Bursa, Turkey, 2010.

[8] S. H. Eryuruk, F. Kalaoghu, The effects of different amounts of lubricant application on the sewing thread performance properties, Textile Research Journal. 80(2010), 1232-1242.

[9] A. Gurada, E. Yukseltan, B. M. Kaplangiray, M. Kanik, The effect of various lubricants on the friction properties of sewing threads, Textile Research Journal. 83(2013), 1273-1282.

[10] B. Meric and A. Durmaz. Effect of thread structure and lubrication ratio on seam properties, Indian journal of Fibre \& Textile Research. 30(2005), 273-277.

[11] V. K. Midha, R. Chattopadhyay, V. K. Kothari, and A. Mukhopadhyay, Effect of process and machine parameters on changes in tensile properties of threads during high-speed industrial sewing, Textile Research journal. 79(2009), 1155-1167.

[12] V. K. Midha and S. Verma, Effect of lubrication on the changes in tenacity of cotton sewing threads at different stages of sewing, Textile Bioengineering and Informatics Symposium Proceedings, 2018, 496-502. 